\title{
Evaluación del uso correcto de las heparinas de bajo peso molecular (HBPM) en pacientes con indicación de anticoagulación, evaluando el ajuste según peso,edad y depuración de creatinina
}

\author{
Assessment of the correct use of low-molecular-weight \\ heparins (LMWHs) in patients with indication for \\ anticoagulant therapy taking into account adjustment to \\ body weight, age, and creatinine clearance
}

DARÍO LONDOÑo TRUJILLO, MD(1); ANDRÉS GARZÓN FORERO, MD(2)

\section{Resumen}

OBJETIVOS: analizar la aplicación adecuada de las guías de anticoagulación y el ajuste según peso, edad y depuración de creatinina en los pacientes adultos hospitalizados en el Hospital Universitario San Ignacio.

METODOLOGÍA: estudio descriptivo en el cual se revisaron 629 historias de pacientes hospitalizados en el Hospital Universitario de San Ignacio durante el periodo 2009 y 2010 , quienes recibieron heparinas de bajo peso molecular según las indicaciones individuales. Se dividieron en 4 grupos según el medicamento recibido; grupo 1: nadroparina de 0,6 con 10 pacientes; grupo 2: nadroparina 0,3 con 18 pacientes; grupo 3: dalteparina de $2.500 \mathrm{mg}$ con 183 pacientes; y grupo 4: enoxaparina de $80 \mathrm{mg}$ con 418 pacientes.

RESULTADOS: se ajustaron únicamente el $17 \%$ contra el $83 \%$ de los pacientes. Las causas en orden de frecuencia fueron peso, seguido por función renal, y cirugía mayor. En el grupo 2 no se ajustaron el $17 \%$ contra el $83 \%$, de los que debieron haberse ajustado principalmente por peso, seguido por cirugía mayor, función renal, edad y diagnóstico oncológico. En el grupo 3 se ajustó el 14\% contra el 86\% que debieron ajustarse por ser oncológicos, seguido por depuración de creatinina, cirugía mayor, edad y peso. En el grupo 4 se ajustaron el $43 \%$ contra el $57 \%$ que debieron ajustarse por cirugía mayor, seguidos por depuración de creatinina, oncológicos, edad y peso.

CONCLUSIONES: se deben tener en cuenta las variables descritas para ajustar las dosis de heparinas de bajo peso molecular en los pacientes hospitalizados, ya que aumenta el riesgo de enfermedad trombo-embólica venosa.

Palabras clave: anticoagulación, edad, peso, oncología, cirugía mayor.

\begin{abstract}
OBJECTIVES: The proper application of the guidelines for anticoagulation and adjustment according to weight, age and creatinine clearance in adult patients hospitalized in HUSI.

METHODOLOGY: Descriptive study in which 629 were reviewed records of patients hospitalized at the Hospital Universitario de San Ignacio during the period between 2009 and 2010, received individual LMWH as directed. They were divided into groups according
\end{abstract}

${ }^{(1)}$ Médico internista, especialista en Neumología, Pontificia Universidad Javeriana, Epidemiólogo Clínico, Universidad de Mc Master, Canadá.

${ }^{(2)}$ Médico residente de tercer año en Medicina Interna. Pontificia Universidad Javeriana. Correspondencia: Darío Londoño. Correo electrónico: dario.londono@gmail.com Recibido: 12-09-2013. Aceptado: 25-12-2013. 
to the drug received Nadroparin The first group was of 0.6 with 10 patients (Group 1), Nadroparin 0.3 with 18 patients (Group 2), dalteparin 2500 with 183 patients (Group 3), enoxaparin $80 \mathrm{mg}$ with 418 patients (Group 4).

RESULTS: Adjusted only $17 \%$ against $83 \%$ of patients who should have adjusted the causes were weight, followed by renal function, and major surgery. In group 2 did not meet the $17 \%$ against $83 \%$ of which should have been adjusted mainly by weight, followed by major surgery, renal function, age and cancer. In group 3 was adjusted to $14 \%$ against $86 \%$ were due to be adjusted for cancer, followed by creatinine clearance, major surgery, age and finally by weight. In group 4 were adjusted to $43 \%$ against $57 \%$ were due primarily to adjust for major surgery, followed by creatinine clearance, oncology, followed by age and weight.

CONCLUSIONS: It must take into account the variables described for adjusting the dose of LMWH in hospitalized patients because it increases the risk of thrombo-embolic venous disease.

Keywords: anticoagulation, age, weight, oncology, surgery.

\section{Introducción}

Se han detectado deficiencias en la formulación de las heparinas de bajo peso molecular (HBPM), al no ajustarlas según el peso del paciente, no disminuir la dosis en los pacientes mayores de 75 años y no ajustarlas a la depuración de creatinina, en los pacientes adultos mayores de 18 años, en los diferentes servicios del Hospital Universitario San Ignacio (HUSI). Estos datos se obtuvieron mediante análisis de la formulación y seguimiento desde el servicio de urgencias, hasta el servicio de hospitalización. Se observó que los pacientes estuvieron expuestos a riesgos, complicaciones y efectos adversos, en especial debido a falta de ajuste de las dosis según características individuales como peso, edad y función renal.

\section{Marco teórico}

La enfermedad trombo-embólica venosa hace referencia a la condición en la que un coágulo migra de un sistema venoso a un órgano, comprometiéndolo en su vasculatura. La mayoría de estos coágulos procede del sistema venoso profundo en miembros inferiores y miembros superiores. A nivel clínico, tienen una correlación entre la trombosis venosa profunda y la tromboembolia pulmonar, pero en otros casos tienen inicio a nivel de los vasos pulmonares secundarios a condiciones del individuo como hipertensión pulmonar primaria, en la que pueden presentar clínica sugestiva de embolia crónica, la cual puede tardar de semanas a meses (1).

En los Estados Unidos se reporta una incidencia de 100.000 casos por año, aunque se sospecha que haya un número mayor debido a subregistro; en

\section{¿Qué se conocía del tema?}

Las guías aceptadas de anticoagulación, recomiendan el ajuste de las dosis según factores como edad, peso, función renal y embarazo entre otras. Varios reportes señalan que dicha recomendación no es tenida con frecuencia.

\section{¿Qué muestra el estudio?}

En un centro de referencia de Bogotá, la frecuencia del ajuste de la dosis de heparinas de bajos peso molecular fue baja, como se ha reportado previamente. Se debe tener en cuenta que el no tener en cuenta estos factores, podría asociarse con eventos adversos secundarios ya sea por dosis elevadas o subterapéuticas.

cuanto a la enfermedad trombo-embólica venosa, ocurre en alrededor de 630.000 pacientes, con una mortalidad de 200.000 por año en la cual la mayoría no fueron diagnosticados (1).

La fisiopatología de la formación de trombos se origina en donde el flujo venoso es turbulento, en una bifurcación o detrás de las válvulas venosas si las posee, y estos son sintomáticos cuando la producción del trombo excede la velocidad de organización, produciendo la ruptura $\mathrm{y}$, por ende, la migración del coágulo. Se pueden originar en los vasos pélvicos en situaciones como el embarazo, por tromboflebitis pélvicas, enfermedad prostática $\mathrm{o}$ cirugía reciente, o bien ser secundarios al implante de dispositivos intravasculares como catéteres $(1,2)$.

Entre los factores de riesgo para tromboembolia venosa, figura la tríada de Virchow en la que se menciona la estasis venosa, la hipercoagulabilidad y la lesión endotelial. Los grupos de pacientes quirúrgicos de Ortopedia, Neurocirugía, Ginecología y Urología son los de mayor riesgo, al igual que los traumas en especial de cabeza, columna y pélvico. 
No solo se incluyen los pacientes intra-hospitalarios, sino aquellos que han viajado en avión durante más de 8 horas o 5.000 millas. Así mismo, se incluyen las mujeres embarazadas mayores de 40 años, las mujeres que planifican con anticonceptivos orales o aquellas con indicación de terapia de reemplazo hormonal (2).

De otra parte, hay múltiples factores de riesgo individuales como la obesidad, definida como un índice de masa corporal mayor de $29 \mathrm{~kg} / \mathrm{m} 2$, que se convierten en un factor para embolia venosa, ya que puede ir de la mano del síndrome metabólico (hipertensión, obesidad abdominal e intolerancia a los carbohidratos) (3).

De igual forma, la edad se convierte en uno de los factores de riesgo que se asume a las comorbilidades que se van adquiriendo con los años, aumentando el riesgo 25 veces en pacientes mayores de 70 años, hecho que genera una alta mortalidad por esta causa. Dentro de estas comorbilidades se encuentra el cáncer que en su orden, pulmón, páncreas, seno, próstata, estomago, colo-rectal y génito-urinario, son los más frecuentes e incrementan el riesgo de cuatro a ocho veces (4).

Ser paciente renal crónico cuenta como factor de riesgo, en especial cuando debutan con síndrome nefrótico dado que tienen el $40 \%$ de riesgo de generar tromboembolia venosa, con presentaciones poco frecuentes como los senos venosos cerebrales o trombosis arteriales, generados por cambios cuantitativos o alteración en las funciones de los factores de coagulación, disminución de la actividad fibrinolítica, hiperreactividad plaquetaria y aumento en la viscosidad sanguínea. Por tanto, tienen indicación de anticoagulación con heparinas no fraccionadas o fraccionadas con cambio posterior a terapia oral con una duración de seis meses. Así mismo, se debe adaptar la dosis de heparinas teniendo en cuenta la tasa de filtración glomerular puesto que se ha descrito resistencia a la heparina sódica debido al descenso en los niveles de anti-trombina III, por lo cual debe ajustarse según los niveles de PTT activado $(1,5)$.

Patologías instauradas con factores de riesgo en común, como síndromes coronarios agudos, entre éstos el infarto agudo del miocardio con o sin elevación del segmento ST y la angina inestable, poseen una fisiopatología común (la ruptura de la placa), la erosión, sumada a trombosis intracoronaria, que requieren manejo con anticoagulación inicialmente con heparinas no fraccionadas; sin embargo, debido a eventos adversos como la trombocitopenia y el riesgo de sangrado, hoy estas se utilizan con menor frecuencia y en cambio se ha pasado al uso de las HBPM dado que sus efectos anticoagulantes pueden predecirse con mayor facilidad, no requieren monitoreo en el laboratorio, ostentan bajas tasas de trombocitopenia y son de aplicación subcutánea $(6,7)$.

Teniendo en cuenta los síndromes coronarios en mención se han llevado a cabo estudios que comparan el uso de la terapia de reperfusión con trombólisis y enoxaparina en el tratamiento del infarto agudo de miocardio (estudio ExTRACT) y trombólisis en infarto agudo del miocardio (estudio TIMI 25). En estos se comparó el uso de enoxaparina vs. heparina no fraccionada en 20.506 pacientes con infarto del miocardio con elevación del ST, que recibieron terapia fibrinolítica, con manejo con heparina no fraccionada por 48 horas y enoxaparina durante la hospitalización con una reducción absoluta del 2,1\% a treinta días de seguimiento. Entre tanto, en el estudio OASIS-5 se comparó el uso de fondaparinux con enoxaparina en un grupo de 20.078 pacientes y se obtuvieron resultados similares en cuanto a mortalidad, infarto del miocardio e isquemia refractaria, así como una disminución en el riesgo de sangrado en los pacientes que recibieron manejo con fondaparinux, motivo por el cual en el caso de los pacientes con eventos coronarios agudos se ha demostrado ampliamente la seguridad y la disminución del riesgo de sangrado, al igual que la trombocitopenia con el uso de enoxaparina, mientras que en los pacientes con riesgo de sangrado no está indicado el uso de fondaparinux en el contexto del paciente con evento coronario agudo (7).

Con el advenimiento de las últimas tecnologías se desarrollaron las heparinas de bajo peso molecular, que en comparación con la heparina no fraccionada presentan ventajas notables como: vida media más prolongada, anticoagulación terapéutica más temprana, no requerimiento de monitorización de efectos anticoagulantes, reducción de sangrados 
mayores y mayor evidencia y estudios clínicos para enoxaparina en relación con las demás heparinas disponibles en el mercado. En general, no requieren monitorización, pero en caso de necesitarla se pueden solicitar niveles de factor $\mathrm{X}$ activado. Son útiles en pacientes con anticuerpos antifosfolípidos o con anticoagulantes ajenos circulantes; peso mayor de 150 kilos o menores de 40 kilos, depuración de creatinina menores de $30 \mathrm{~mL} / \mathrm{min}$, embarazo, sangrados no explicados o eventos trombóticos nuevos durante la terapia. El rango terapéutico debe estar entre 0,6 a $1 \mathrm{U} / \mathrm{mL}$ y para la profilaxis entre 0,1 a $0,3 \mathrm{U} / \mathrm{mL}(5)$.

\section{Métodos}

Estudio descriptivo en el que se evaluaron pacientes con diferentes variables, asignación por género, edad mayor de 18 años y ajuste si presentaba más de 75 años, depuración de creatinina menor de $30 \mathrm{~mL} / \mathrm{min}$, cirugía mayor definida como duración mayor de 30 minutos, obesidad dada por Índice de masa corporal mayor de $30 \mathrm{~kg} / \mathrm{m} 2$ y peso mayor de $110 \mathrm{~kg}$. De todas las historias clínicas revisadas, 151 (24\%) cumplieron los criterios equivalentes. En el grupo 1 el $20 \%$, en el 2 el $50 \%$, en el 3 el $24,5 \%$ y en el 4 el 22,7\%. El principal problema encontrado para el análisis fue el sub-registro en el peso y en la talla.

\section{Resultados}

La edad promedio fue de 64 años y desviación estándar (DE) de $\pm 17,7$ para el grupo 1, de 56 años y DE de $\pm 20,9$ para el grupo 2, de 57 años y DE de $\pm 20,7$ para el grupo 3 , y de 58 años y $\mathrm{DE}$ de $\pm 14,7$ para el grupo 4. Se evidenció homogeneidad para los cuatro grupos.

La talla promedio en el grupo 1 fue de $168 \mathrm{~cm}$ con DE de $\pm 4,9 \mathrm{~cm}$, en el grupo 2 fue de 163 con DE de $\pm 8,2 \mathrm{~cm}$, en el grupo 3 fue de $161 \mathrm{~cm}$ y DE de \pm 10,1 , mientras que para el grupo 4 fue de $167 \mathrm{~cm} \mathrm{y}$ $\mathrm{DE}$ de $\pm 9,11$, lo cual hace similares a los grupos $1 \mathrm{y}$ 4 , у 2 у 3 , respectivamente.

El peso promedio para el grupo 1 fue de $67 \mathrm{~kg}$ con $\mathrm{DE}$ de $\pm 20 \mathrm{~kg}$, para el grupo 2 fue de $67,7 \mathrm{y}$ $\mathrm{DE}$ de $\pm 22,87$, para el grupo 3 fue de $64,1 \mathrm{~kg}$ y DE de $\pm 9,41$ y para el grupo 4 de 76,5 y DE de $\pm 14,6$ $\mathrm{kg}$, con homogeneidad en el grupo 1, 2 y 3, pero diferencia en el grupo 4, en el que hubo un promedio de peso mayor, con un rango de variabilidad amplio en los primeros dos pero estrecho en los últimos dos grupos.

El índice de masa corporal en el grupo 1 fue de 27,3 con DEE de 2,87, en el grupo 2 fue de 25,04 y De de $\pm 3,59$. En el grupo 2 fue de 25 y DE de $\pm 3,59$; en el grupo 3 fue de 23,06 y $\mathrm{DE}$ de $\pm 3,79$, mientras que $n$ el grupo 4, fue de 27,14 y DE de \pm 5,7. Así, pues, se evidenció un índice de masa corporal dentro del rango de sobrepeso, similar en los cuatro grupos, con una variación estrecha en los primeros y más amplia en el último.

En lo que respecta al género, el $61 \%$ fueron hombres y el 39\% mujeres, relacionado con la enfermedad coronaria como indicación para controlar los factores de riesgo cardiovascular dado el promedio de edad asociado con la patología por la cual fueron tratados.

Otra de las variables evaluadas fue la depuración de creatinina como valor referente en hombres hasta 1.2 y en mujeres hasta 1.0, en el grupo 1 el promedio del valor de creatinina fue de 1.42 con una DS d de $+/-0,96$. En el grupo 2 el valor de creatinina fue de 1.07 y una DS de $+/-0,82$, en el grupo 3 el valor de creatinina fue de 1,62 con una DS de $+/-2,14$, en el Grupo 4 el valor de creatinina fue de 1,36 con una DS de $+/-1,77$. Como resultado evidenciamos que la mayoría están fuera del valor normal lo cual requiere el calculo de la depuración de creatinina para evaluar si requiere el ajuste de las heparinas de bajo peso molecular.

En el cálculo de la Depuración de creatinina el Grupo 1 tuvo un promedio de 59,5 $\mathrm{ml} / \mathrm{min}$ con una DS de +/- 42,92, Para el grupo 2 29,9 $\mathrm{ml} / \mathrm{min}$ con una DS de $+/-13,13$. En el grupo 3 el valor fue de 24,6 con una DS de +/- 15,62 y en el grupo 429,50 con una DS de $+/-15,39$. Por los datos obtenidos previamente mas de la mitad de los pacientes requieren ajuste de la dosis por presentar depuraciones de creatinina menores de $30 \mathrm{ml} / \mathrm{min}$ y por el rango de las desviaciones estándar mas del $50 \%$ están por debajo de este punto de corte.

Los pacientes que debieron estar anticoagulados profilácticamente por ser sometidos a cirugía mayor, 
en el grupo 1 el $40 \%$ de los pacientes requirieron ajuste de las mismas contra el $60 \%$ que no las requería. En el Grupo 2el 39\% contra $61 \%$ de los pacientes requerían estar anticoagulados profilácticamente. En el grupo 3 el $28 \%$ contra el $72 \%$ que deberían estar anticoagulados. En el grupo $428 \%$ contra el $72 \%$ que requerían anticoagulación profiláctica. Es decir aproximadamente el $30 \%$ de los pacientes que son llevados a cirugía son cirugías mayores y que requieren anticoagulación profiláctica de entrada al menos por esta indicación.

Los pacientes con patología oncológica también fueron evaluados, en el grupo 1 no se encontraron pacientes oncológicos, en el grupo 2 17\% tenían patología oncológica contra $83 \%$ que no la tenía, en el grupo $342 \%$ contra $58 \%$ tenían patología oncológica y en el grupo $447 \%$ contra 53\% tenían patología oncológica. Con estos resultados hay mayor población de pacientes oncológicos en el grupo 4 que recibieron heparinas de bajo peso molecular con respecto a los grupos 1,2 y 3, debido a que eran del grupo de Enoxaparina de $80 \mathrm{mg}$, relacionado con el riesgo de complicaciones embolicas en este grupo por su diagnostico de base oncológico.

\section{Discusión}

Teniendo en cuenta los resultados previos en el grupo 1 se ajustaron únicamente el $17 \%$ contra el $83 \%$ de los que se debieron haber ajustado principalmente por peso, seguido por función renal, y cirugía mayor. En el grupo 2 no se ajustaron el 17\% contra el $83 \%$, de los que se debieron haber ajustado principalmente por peso, seguido por cirugía mayor, función renal, edad y oncológico. En el grupo 3 se ajusto el $14 \%$ contra el $86 \%$ se debieron ajustar por ser oncológicos, seguido por depuración de creatinina, cirugía mayor, edad y por ultimo por peso. En el grupo 4 se ajustaron el $43 \%$ contra el $57 \%$ se debieron ajustar principalmente por cirugía mayor, seguidos por depuración de creatinina, oncológicos, por edad y peso.

\section{Conclusiones}

Se deben usar racionalmente las heparinas de bajo peso molecular con el fin de disminuir el riesgo de enfermedad trombo-embolica venosa en los pacientes hospitalizados.

Es importante tener en cuenta las variables mencionadas en el análisis realizado, ya que según estos factores pueden presentarse eventos adversos secundarios al no ajuste de las mismas ya sea por dosis elevadas o subterapéuticas.

Teniendo en cuenta los resultados anteriores hay que realizar una campaña de socialización y de re educación hacia todas las especialidades medicas y quirúrgicas en especial los resultados arrojados por el grupo 4 que es la molécula mas utilizada a nivel hospitalario y ambulatorio.

Teniendo una herramienta de fácil registro como es la historia clínica, se debe promover el diligenciamiento y la accesibilidad a los registros de peso y talla que son muy importantes, para que no solo el medico sea el único que tenga acceso al registro del peso y de la talla, sino el grupo de enfermería y grupo de terapia.

Realizando estos cambios y mejorando el objetivo terapéutico individualizando cada una de las variables analizadas durante el estudio, cumpliéndolas y ajustando las dosis en cada uno de nuestros pacientes podríamos mejorar nuestros casos de enfermedad trombo-embólica venosa en nuestros pacientes del HUSI.

\section{Referencias}

1. Fishman Alfred, Elias JA, Grippi MA, Senior RM, et al. Fishman`s Pulmonary Diseases and Disorders, Fourth Edition Mc Graw Hill 1423-1448.

2. Kucher N, Goldhaber SZ: Risk stratification of acute pulmonaryembolism. Semin Thromb Hemost 32:838-847,2006.

3. Raschke RA, Reilly BM, Guidry JR, et al.: The weight based heparin dosing nomogram compared with a "standard care" nomogram: A randomized controlled study. Ann Intern Med 119:874-881,1993.

4. Stein PD,Hull RD, Kayali F, et al.: Venous thromboembolismaccording to age: The impact of an aging population. Arch Intern Med 164:2260-2265, 2004.

5. R. Alcázar, J. M. López Gómez y F. Rivera.: Manejo general del síndrome nefrótico. NEFROLOGÍA. Volumen 27. Suplemento 2. 2007

6. Roy PM, Colombet I, Durieux P, et al.: Systematic review and metaanalysis of strategies for the diagnosis of suspectedpulmonary embolism. Br Med J 331:259-268, 2005.

7. Raymond J. Gibbons, M.D., and Valentin Fuster, M.D., Ph.D.: Therapy for Patients with Acute Coronary Syndromes - New Opportunities. N Engl J med 354;14 April 6, 2006. 